\title{
LOS DERECHOS LINGÜÍSTICOS Y LA EDUCACIÓN EN LENGUAS INDÍGENAS EN LA ENCRUCIJADA DEL DESARROLLO SOSTENIBLE
}

\author{
LINGUISTIC RIGHTS AND EDUCATION IN INDIGENOUS \\ LANGUAGES AL THE CROSSROADS OF SUSTAINABLE \\ DEVELOPMENT
}

\begin{abstract}
DERECHOS LINGÜISTICOS NISQATA ÑAWPAQ RIMARIYKUNA YACHACHIYMANTAWAN CHAY ALLIN KAWSAY ÑAUPAQMAN PURINAMPAQ ${ }^{(1)}$
\end{abstract}

Ines Ma Garcia-Azkoaga ${ }^{(2)}$

Universidad del País Vasco, España

Resumen: La diversidad lingüística es parte del patrimonio inmaterial del mundo, pero no todas las lenguas gozan del mismo estatus ni del mismo reconocimiento. Junto a las grandes lenguas mayoritarias existen numerosas lenguas indígenas minimizadas a las que apenas se les presta atención, y a cuyos hablantes se les priva de algunos derechos básicos como el de ser educados en su lengua autóctona. Este artículo aborda la dimensión interactiva del lenguaje como parte de la actividad humana, y la lengua materna como un derecho de los hablantes reconocido por la UNESCO; luego pone atención en el papel que juega la diversidad lingüística en los Objetivos de Desarrollo Sostenible; también recalca la necesidad de favorecer una educación plurilingüe articulada en torno a la lengua materna.

Palabras clave: Derechos lingüísticos, diversidad lingüística, educación plurilingüe, lenguas indígenas, lenguas y desarrollo sostenible.

Abstract: Linguistic diversity is part of the world's intangible heritage. But not all languages enjoy the same status or recognition. Alongside the great majority

(1) Traducción: Alejandra C. Ninahuaman Quispe, Kilmer Ttito Quispe (Lengua Quechua / Cusco Qollao).

(2) Cátedra Unesco de Patrimonio Lingüístico Mundial. Profesora de la Universidad del País Vasco (UPV/EHU). ines.garciaazkoaga@ehu.eus 
languages, there are many indigenous and minority languages that are barely noticed, and whose speakers are deprived of some basic rights such as education in their native language. This contribution address to the interactive dimension of language as part of human activity, and the mother tongue as a speaker's right recognized by UNESCO; then it turns the attention to the role played by linguistic diversity in the Sustainable Development Goals to underline its importance for such development; It also stress the need to foster mother-tongue-based multilingual education.

Key Words: Linguistic rights, linguistic diversity, multilingual education, indigenous languages, languages and sustainable development.

Ñujñu: Llapa rimakuykuna yuyayninchispi kan, icha mana lliw rimaykunaqa mana reqsisqachu kashan. Hoq rimakuykuna ashqa runakuna rimanku, chay rimakuykunaqa allin reqsisqa; hoqkunataq mana ihirinkuchu ni reqsisqachu kan, ankiy chikaq rimaqkuna mana qonkuchu derechos básicos nisqata, manan yachachinkuchu rimayninkupi. Chayna kay qelqay rimarin imaynata rimayninchis may chaniyuq noqanchispaq, chaynaqa reqsisqa kashan sapan ayllu rimayninkupi kay UNESCO nisqapi, chaymanta qawarin imaynata chay lliw rimakuykuna allinta ñawpaqman puririchin, aqnapis yuyarichin imaynata kanan yachachiy sapan rimayninchispi.

Kichana rimaykuna: Derechos lingüísticos, diversidad lingüística, educación plurilingüe, lenguas indígenas, lenguas y desarrollo sostenible. 


\section{Introducción}

Una de las principales capacidades que tiene el ser humano es el lenguaje. A través del lenguaje no solo nos comunicamos, también interactuamos con los demás, aprendemos, regulamos comportamientos, transmitimos y desarrollamos conocimientos, nos divertimos, imaginamos y representamos el mundo. Es la forma en que nos relacionamos, pero es sobre todo un hecho social cuyo uso está ligado al contexto y a los avatares socio-históricos que le dotan de significado. El lenguaje es, en definitiva, una actividad estrechamente ligada con el contexto. Esa particularidad es común sea cual sea la lengua o idioma que utilicemos para comunicarnos, pues el funcionamiento comunicativo reposa sobre los mismos principios: un locutor con un objetivo comunicativo, una situación de comunicación a la que debe adaptar su mensaje, un contenido al que debe dar forma, unos parámetros físicos y espaciales, unos condicionamientos sociales y, por supuesto, un código, una lengua en la que transcribir el mensaje.

Aunque el funcionamiento el lenguaje se puede considerar igual para todos los humanos, sus manifestaciones se traducen en lenguas o idiomas concretos que reflejan las relaciones y saberes de la comunidad en la que se habla, su historia y su tradición, de ahí proviene la diversidad lingüística y cultural. De hecho, en el mundo se contabilizan al menos 6.700 lenguas diferentes según los estudios realizados por expertos como Martí et al. (2006), de las cuales más de 2.600 son vulnerables o están en peligro de desaparición; además, un 33\% de las lenguas del mundo no está presente en la educación, dicho de otra manera, los hablantes de esas lenguas no tienen acceso a una escolarización en lengua indígena o autóctona, que es su lengua materna.

Frente a las seis lenguas más habladas del mundo (chino, español, inglés, hindi, árabe y portugués), existen otras muchas que tienen muy pocos hablantes, como por ejemplo el islandés, pero que hoy por hoy son la lengua mayoritaria del país. Esas lenguas, aunque minoritarias, tienen una ventaja de la que carecen muchas lenguas indígenas, autóctonas u originarias (según las diferentes denominaciones que podemos utilizar), y es que son lenguas que tienen un reconocimiento oficial en los países en los que se utiliza, aun cuando en nuestros días sufren la presión de lenguas extranjeras como el inglés.

Contrariamente, hay otras lenguas que no solo son minoritarias respecto a las grandes lenguas que se hablan en el mundo, sino que, además, están minorizadas, es decir, no cuentan con un estatus de reconocimiento similar al de las lenguas mayoritarias. Es el caso de muchas lenguas originarias o indígenas sometidas a procesos de colonización que desplazaron el uso y la oficialidad de esa lengua en su propio territorio y que generaron, incluso, procesos de aculturación. Es el caso del francés y del inglés en África o del español en América Latina. Tampoco 
las lenguas europeas fueron inmunes al contacto con las lenguas mayoritarias y a las políticas lingüísticas monolingües ejercidas por los Estados.

A pesar de la declaración de las Naciones Unidas (1963) sobre el uso de la lengua materna o indígena ${ }^{(3)}$ en la educación, las consecuencias de las políticas monolingües perduran aún en nuestros días impidiendo el desarrollo lingüístico y cultural de muchas comunidades indígenas y lenguas minorizadas en todo el mundo. Es por eso que la Organización de las Naciones Unidas (ONU) proclamó el 2019 como Año Internacional de las Lenguas Indígenas, cuya coordinación estuvo a cargo de la UNESCO. Para conmemorarlo, se realizaron eventos de todo tipo a lo largo del mundo, desde congresos y publicaciones académicas especializadas hasta jornadas y acciones de difusión y divulgación, como exposiciones, conciertos, juegos, animaciones, documentales, escenificaciones, etc. ${ }^{(4)}$. Pero lo que realmente se pretende no es solo conmemorar el año sino también dar pasos adelante en la consecución de los derechos de los pueblos indígenas, tal como fueron reconocidos en la Declaración de las Naciones Unidas sobre los derechos de los pueblos indígenas $(2007)^{(5)}$, que tiene por objeto la protección de esos pueblos y la salvaguarda de sus derechos para preservar su cultura, tradiciones e instituciones. En dicha Declaración no está ausente la reivindicación lingüística; en sus artículos 13 al 16 podemos leer, concretamente, como aparecen plasmados el derecho a la revitalización lingüística $y$, entre otros, el derecho a una educación en su lengua propia:

\section{"Artículo 13}

1. Los pueblos indígenas tienen derecho a revitalizar, utilizar, fomentar y transmitir a las generaciones futuras sus historias, idiomas, tradiciones orales, filosofías, sistemas de escritura y literaturas, y a atribuir nombres a sus comunidades, lugares y personas, así como a mantenerlos.

2. Los Estados adoptarán medidas eficaces para asegurar la protección de ese derecho y también para asegurar que los pueblos indígenas puedan entender y hacerse entender en las actuaciones políticas, jurídicas y administrativas, proporcionando para ello, cuando sea necesario, servicios de interpretación u otros medios adecuados.

\section{Artículo 14}

1. Los pueblos indígenas tienen derecho a establecer y controlar sus sistemas e instituciones docentes que impartan educación en sus propios idiomas, en consonancia con sus métodos culturales de enseñanza y aprendizaje.

(3) En el marco de una educación plurilingüe, cuando decimos lengua "propia" podríamos decir "originaria", "materna", "indígena", "autóctona"...; es un concepto que viene adquiriendo diferentes denominaciones con el transcurso de los años.

(4) https://es.iyil2019.org/events/

(5) https://www.un.org/development/desa/indigenous-peoples-es/declaracion-sobre-los-derechos-de-los-pueblos-indigenas.html 
2. Los indígenas, en particular los niños, tienen derecho a todos los niveles y formas de educación del Estado sin discriminación.

3. Los Estados adoptarán medidas eficaces, conjuntamente con los pueblos indígenas, para que las personas indígenas, en particular los niños, incluidos los que viven fuera de sus comunidades, tengan acceso, cuando sea posible, a la educación en su propia cultura y en su propio idioma.

\section{[...]}

\section{Artículo 16}

1. Los pueblos indígenas tienen derecho a establecer sus propios medios de información en sus propios idiomas y a acceder a todos los demás medios de información no indígenas sin discriminación." (ONU, 2007: 7).

Por otra parte, en sus artículos 28 y 29, el Convenio sobre Pueblos Indígenas y Tribales de la Organización Internacional del Trabajo (1989) hace alusión a los derechos lingüísticos en estos términos:

\section{"Artículo 28}

1. Siempre que sea viable, deberá enseñarse a los niños de los pueblos interesados a leer y a escribir en su propia lengua indígena o en la lengua que más comúnmente se hable en el grupo a que pertenezcan. Cuando ello no sea viable, las autoridades competentes deberán celebrar consultas con esos pueblos con miras a la adopción de medidas que permitan alcanzar este objetivo.

2. Deberán tomarse medidas adecuadas para asegurar que esos pueblos tengan la oportunidad de llegar a dominar la lengua nacional o una de las lenguas oficiales del país.

3. Deberán adoptarse disposiciones para preservar las lenguas indígenas de los pueblos interesados y promover el desarrollo y la práctica de las mismas.

\section{Artículo 29}

1. Un objetivo de la educación de los niños de los pueblos interesados deberá ser impartirles conocimientos generales y aptitudes que les ayuden a participar plenamente y en pie de igualdad en la vida de su propia comunidad y en la de la comunidad nacional." (OIT, 1989: pp. 57-58).

El derecho a usar la lengua propia está también recogido en la Resolución aprobada por la ONU en 2014 (Documento final sobre la Conferencia Mundial sobre los Pueblos Indígenas): 
"Nos comprometemos a promover el derecho de todos los niños indígenas, en común con los demás miembros de su grupo, a tener su propia vida cultural, a profesar y practicar su propia religión y a emplear su propio idioma." (ONU, 2014).

A pesar de todos los esfuerzos, normas y declaraciones, la pérdida de las lenguas en el mundo no se ha revertido, sino que se ha agudizado, se estima que se pierde una lengua cada dos semanas. Cada lengua que desaparece se lleva consigo un patrimonio cultural irrecuperable, por eso es importante la conmemoración del Año Internacional de las Lenguas Indígenas, pero más aún que las iniciativas puestas en marcha tengan una continuidad en el tiempo.

Una de las propuestas interesantes que se suma a la salvaguarda de la diversidad lingüística y cultural es la Declaración Americana sobre los Derechos de los Pueblos Indígenas (2016). En su preámbulo el documento hace hincapié en la idiosincrasia de las culturas indígenas y reconoce la contribución al desarrollo sostenible de las prácticas tradicionales de esos pueblos; también subraya la importancia de eliminar cualquier tipo de discriminación. En la formulación de sus artículos, hace referencia explícita a las lenguas, tanto en lo que se refiere al ámbito de aplicación: "Los Estados reconocen y respetan el carácter pluricultural y multilingüe de los pueblos indígenas, quienes forman parte integral de sus sociedades." (OAS, 2016: 3), como a los derechos humanos y colectivos:

"Los pueblos indígenas tienen derechos colectivos indispensables para su existencia, bienestar y desarrollo integral como pueblos. En este sentido, los Estados reconocen y respetan, el derecho de los pueblos indígenas a su actuar colectivo; a sus sistemas o instituciones jurídicos, sociales, políticos y económicos; a sus propias culturas; a profesar y practicar sus creencias espirituales; a usar sus propias lenguas e idiomas; y a sus tierras, territorios y recursos. Los Estados promoverán con la participación plena y efectiva de los pueblos indígenas la coexistencia armónica de los derechos y sistemas de los grupos poblacionales y culturas." (OAS, 2016: 4).

Tampoco olvida la importancia de que las lenguas indígenas estén presentes en todos los ámbitos de la vida, incluso en la educación:

"Los pueblos indígenas tienen derecho a establecer y controlar sus sistemas e instituciones docentes que impartan educación en sus propios idiomas, en consonancia con sus métodos culturales de enseñanza y aprendizaje." (OAS, 2016: 6)

"Los Estados, en conjunto con los pueblos indígenas, adoptarán medidas eficaces para que las personas indígenas, en particular los niños y niñas, que viven fuera de sus comunidades puedan tener acceso a la educación en sus propias lenguas y culturas." (OAS, 2016: 6)

“Los Estados promoverán relaciones interculturales armónicas, asegurando en los sistemas educativos estatales, una currícula con 
contenidos que reflejen la naturaleza pluricultural y multilingüe de sus sociedades y que impulsen el respeto y el conocimiento de las diversas culturas indígenas. Los Estados, en conjunto con los pueblos indígenas, impulsarán la educación intercultural que refleje las cosmovisiones, historias, lenguas, conocimientos, valores, culturas, practicas y formas de vida de dichos pueblos." (OAS, 2016: 6)

No obstante, la propuesta necesita el consenso de todos los Estados y también estrategias de colaboración y trabajo a largo plazo con los pueblos indígenas para implementar medidas que sean eficaces para el desarrollo práctico de los derechos contemplados en esa declaración y en el seguimiento de sus resultados.

\section{Desarrollo sostenible, y diversidad lingüística y cultural}

Además de avanzar en la puesta en práctica de todos los derechos relativos a las comunidades indígenas y a sus lenguas, otro de los objetivos del Año Internacional de las Lenguas Indígenas es el de facilitar que se cumplan los Objetivos de Desarrollo Sostenible al 2030 establecidos por la Organización de Naciones Unidades (ONU).

En septiembre de 2015 la ONU aprobó la Agenda 2030 de los Objetivos de Desarrollo Sostenible (ODS). La agenda establece 17 objetivos dirigidos a conseguir un mundo mejor y más justo, sin embargo, aún cuando existe una pequeña referencia a la educación plurilingüe en el objetivo de la Educación (ODS 4), entre esos ODS no se contempla específicamente la preservación de la diversidad lingüística y el uso normalizado de las lenguas no hegemónicas (llámense autóctonas, minorizadas, minoritarias, indígenas, etc), no se explicita ningún objetivo relativo a la diversidad lingüística y cultural, y tampoco se hace referencia al papel que juegan las lenguas en la consecución de los ODS. Ante esa carencia, desde la Cátedra UNESCO de Patrimonio Lingüístico Mundial de la UPV/EHU se abordó la campaña"17+1" (imagen 1) para sensibilizar, especialmente a la sociedad vasca, sobre la importancia de que el objetivo cultural y lingüístico estuviera también recogido en los ODS. Ese nuevo objetivo (imagen 2) fue plasmado en el panel de los ODS (imagen 3) y en las actividades de sensibilización llevadas a cabo por la Cátedra.

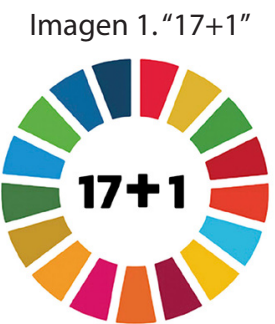

(Fuente: MHOUK ${ }^{(6)}$ )
Imagen 2. Objetivo 18 para los ODS
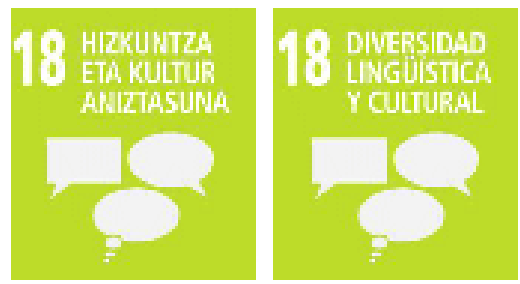

(Fuente: MHOUK)

(6) Cátedra Unesco de Patrimonio Lingüístico Mundial de la UPV/EHU - UPV/EHUko Munduko Hizkuntza Ondarearen Unesco Katedra (MHOUK) 
Imagen 3. Panel de los ODS + Diversidad lingüísica y cultural

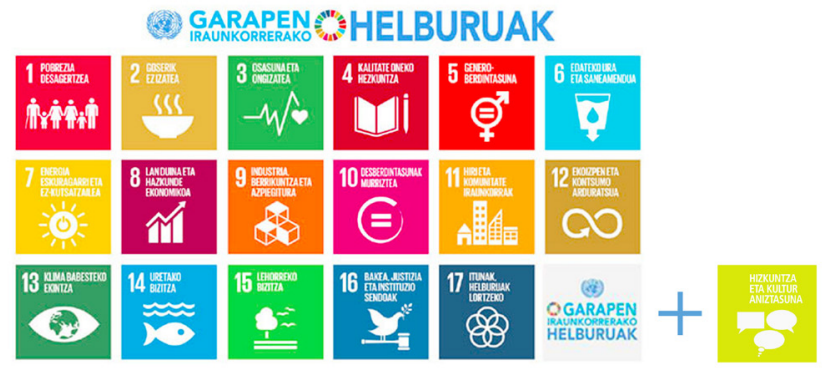

(Fuente: Adaptación realizada por MHOUK)

Además, fruto de la colaboración interdisciplinar con el Instituto de Formación Profesional Easo Politeknikoa de Donostia-San Sebastián, se creó una imagen representativa de la diversidad lingüística (imagen 4), que se incorporó a las acciones de divulgación que se llevaron a cabo.

Imagen 4. Representación de la diversidad lingüística y cultural

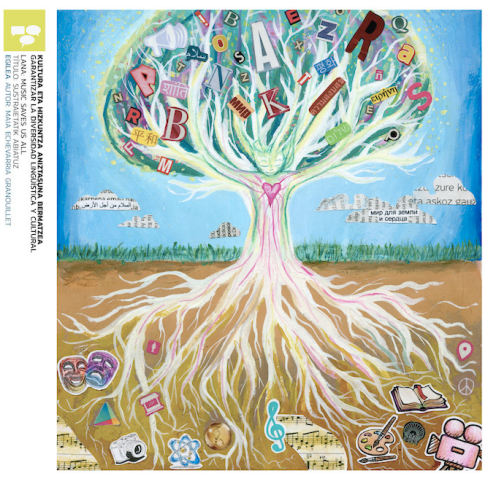

(Fuente: MHOUK)
Imagen 5. El objetivo 18 en la UPV/EHU

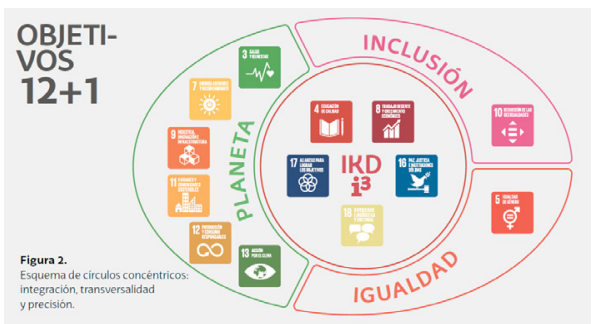

(Fuente: EHU agenda 2030, 2019: p. 7)

El mencionado instituto ha incorporado ese nuevo objetivo como un objetivo transversal en su oferta docente. Gracias a esa iniciativa, también la organización no gubernamental Fundación TAU ha incorporado el objetivo de la diversidad lingüística a sus materiales y acciones de divulgación. Asimismo, la reivindicación "17+1" ha tenido su eco en la Universidad del País Vasco (UPV/EHU), que ha incorporado el nuevo objetivo a los que conforman la agenda 2030 de los Objetivos de Desarrollo Sostenible de dicha universidad (imagen 5). 
Pero también hubieron otras voces que repararon en la importancia que tienen las lenguas para el logro de los ODS. La Asamblea de las Naciones Unidas no ha sido ajena a esa preocupación; a lo largo de los simposios realizados durante estos últimos años ha señalado, por ejemplo, que:

"Solo participando en un discurso colectivo multilingüe, escuchando a los principales interesados de los ODS (aquellos cuyas vidas mejorarían más con su logro), los planos de desarrollo, los planes de acción y las agendas de desarrollo se vuelven inclusivos, equitativos y sostenibles ${ }^{(7)}$ [12]." (Marinotti, 2017: 10).

"Las políticas de desarrollo insensibles al lenguaje amenazan con socavar los esfuerzos educativos, ya que aumentar la asistencia a las escuelas, por ejemplo, no conducirá a mejores resultados educativos si los niños no aprenden realmente" (Marinotti, 2016: 3)

Los objetivos no se entienden si no se tienen en cuenta a las lenguas y serán inalcanzables si esas lenguas no están presentes en la educación. A los ODS se llega a través de la educación y a la educación a través de las lenguas, como decíamos al inicio de este trabajo, pues la actividad verbal es inherente al ser humano y a su actividad general. Es una actividad transversal. Las relaciones entre los individuos y el entorno se materializan a través de la actividad humana y de la actividad verbal, por eso las lenguas deberían de tener un lugar propio en los ODS.

Garantizar la presencia y el reconocimiento de todas las lenguas indígenas y sus culturas es un factor imprescindible, no solo para preservarlas, sino para garantizar también el desarrollo personal y social. La actividad humana y la actividad del lenguaje confluyen en todas las interacciones humanas. La lengua impregna toda la actividad humana y está presente de forma implícita en todos los ODS.

No podemos erradicar la pobreza si en los programas de cooperación no incluimos criterios lingüísticos; no podemos promover la salud y el bienestar si no nos podemos comunicar con los interesados en sus propias lenguas, ¿cómo hacer frente a una epidemia del ébola o a una pandemia mundial como la del Covid-19 sin utilizar la lengua de los pacientes en riesgo?, ¿cómo les hacemos llegar la información que necesitan para la prevención y para sus cuidados?; no podemos proporcionar educación de calidad dejando fuera de la escuela las lenguas autóctonas, ¿cómo evitar el abandono escolar?, ¿cómo garantizar una mínima formación para el mundo laboral?; tampoco podremos reducir las desigualdades ni alcanzar los objetivos de justicia e instituciones sólidas prescindiendo de las lenguas indígenas. En las alianzas para lograr objetivos es esencial que la diversidad

(7) "It is only by engaging in a collective multilingual discourse, by listening to the primary stakeholders of the SDGs (those whose lives would be most improved by their achievement), that development blueprints, action plans and development agendas become inclusive, equitable, and sustainable" (...) "Language-insensitive development policies threaten to undermine educational efforts, since increasing attendance in schools, for example, will not lead to better educational outcomes if children do not actually learn."Traducción del Equipo de Edición de la Revista. 
lingüística y cultural sea tomada en cuenta. Tampoco podemos hacer pedagogía sobre objetivos medioambientales sin tener en cuenta las lenguas y culturas de sus principales destinatarios, y así con todos los ODS.

Consciente de esa diversidad, por ejemplo, el gobierno de Perú está realizando campañas de difusión sobre la Ley Marco de Cambio Climático en las principales lenguas indígenas: quechua en versión oral e impresa, aymara, awajún, asháninka y shipibo-conibo en versión oral. Pero si hay algo fundamental para el desarrollo, es la educación, pero no una educación que invisibilice las lenguas autóctonas sino una educación multilingüe que ayude a desarrollar las capacidades de los alumnos en más de una lengua. No podemos seguir pensando que unas lenguas son mejores que otras o que unas valen más o menos que otras.

Igual que la biodiversidad es esencial para nuestro planeta, la diversidad lingüística forma parte de nuestro ecosistema, y para su pervivencia es necesario salvaguardar las funciones que estas lenguas realizan en todos los ámbitos de uso de su propia comunidad lingüística: socioeconómico, cultural, administrativo, medios de comunicación y de la vida social en general.

Es más, si la Agenda 2030 pretende ser "un plan de acción a favor de las personas, el planeta y la prosperidad" (ONU, 2015) es necesario poner a las personas en el centro de sus acciones, reconociendo, por lo tanto, sus identidades y sus derechos, sin olvidar que detrás de cada lengua hay hablantes, es decir, personas que también tienen unos derechos lingüísticos que deben ser abordados con equidad.

No podemos realizar aquí un análisis exhaustivo de lo que supone la diversidad lingüística y cultural para cada uno de los ODS, pero considerando la importancia que tiene la presencia de las lenguas indígenas en la educación, vamos a poner el foco en ese ámbito.

\section{La revitalización de las lenguas minorizadas y la educación plurilingüe como motor de progreso y desarrollo sostenible}

Es interesante la reflexión que realiza Baztarrika sobre la revitalización lingüística como factor de desarrollo sostenible en un mundo globalizado. El autor toma como ejemplo la doble transformación socioeconómica y sociolingüística que se produjo en la Comunidad Autónoma de Euskadi (CAE) en los años 80 para poner en valor la relación entre "el desarrollo humano sostenible y la preservación de la diversidad lingüística en las sociedades plurilingües"(Baztarrika, 2019: 243). En lo que concierne a la situación de las lenguas y al desarrollo económico, menciona algunos hitos en la sociedad vasca, que merecen ser destacados:

a) El enorme descenso del porcentaje de vascohablantes (del $70 \%$ al $21,9 \%$ ) que se registró en la sociedad vasca entre el siglo XIX y el inicio de la década de los 80 del siglo XX. Retroceso influido, entre otros, por el desprestigio de la lengua, la imposición del castellano como única 
lengua en la educación, y los movimientos migratorios producto de la industrialización.

b) La transformación socioeconómica y sociolingüística llevada a cabo desde 1982, cuando se aprobó la ley de normalización del euskera.

c) El crecimiento del número de vascohablantes desde la puesta en marcha de los planes de revitalización lingüística del euskera, que según los últimos datos disponibles son un $41 \%$ de la población de 20 más años.

Revertir la pérdida de la lengua no es un proceso sencillo, implica un enorme compromiso social. Pero a la vista de los datos que nos ofrece Baztarrika (2019), no hay que menospreciar el valor económico de las lenguas autóctonas minorizadas (llámese, si se prefiere, indígenas), cuyo impacto, en el caso del euskera, se refleja tanto en los ámbitos de la educación y de la administración, como en toda la industria de la lengua y de la cultura, que en su conjunto generaron un $6,3 \%$ del empleo de la CAE en 2015, y una aportación al Producto Interior Bruto (PIB) del 4,2\%.

A esto hay que añadir las posibilidades que ofrece el plurilingüismo en una sociedad en la que el mercado y el consumo están tan presentes, pues el uso de las lenguas autóctonas puede suponer una ventaja frente a los competidores cuando se convierte en una estrategia de marca (ver por ejemplo, el proyecto de política lingüística Emmarca't, 2019).

Los cambios, por supuesto, no solo se deben a las políticas de normalización lingüística, pues como el propio Baztarrika indica, el doble cambio (socioeconómico y sociolingüístico) operado en Euskadi a partir de la década de 1980 es producto de "una estrategia multidimensional propia de un desarrollo humano sostenible" que considera la revitalización de la lengua como "parte constitutiva del gran cambio social" (2019: 255). En definitiva, el autor subraya la vinculación intrínseca entre las dos grandes transformaciones.

En este proceso de revitalización, la educación es, además, un elemento esencial tanto para la recuperación lingüística como para el desarrollo económico. Como recuerda Garcia-Azkoaga (2019), hasta 1978 el español era la única lengua oficial de todo el Estado español. Fue en ese año cuando se aprobó la Constitución española, y en 1979 cuando se aprobó el Estatuto de la Comunidad Autónoma de Euskadi (CAE), reconociéndose en ambos documentos la oficialidad del euskera junto al español. En 1982 se aprobó la cooficialidad de esas lenguas en la Comunidad Foral de Navarra, en la Ley de Restablecimiento del Régimen Foral. Conseguir el estatus de cooficialidad de la lengua vasca junto con el español supuso que a partir de esa fecha se pudiera llevar a cabo un proceso de normalización lingüística en todos los estamentos sociales, pero principalmente en el sistema educativo. En el 
caso de la CAE, el camino fue facilitado por el desarrollo de la Ley de Normalización del Euskera (Gobierno Vasco, 1982) que reconoce la lengua vasca como "lengua propia de la Comunidad Autónoma del País Vasco y el euskera y el castellano como lenguas oficiales en su ámbito territorial" (Gobierno Vasco, BOPV, n 160: 3139). En el terreno de la educación, ello supuso la necesidad de realizar un profundo cambio organizativo y pedagógico para llevar a cabo una educación bilingüe (en realidad, plurilingüe) de calidad que garantizara la transmisión del euskera en aquellas familias que lo tenían como lengua materna o primera, su aprendizaje para aquellos alumnos que tenían como lengua familiar o primera el castellano, y por supuesto, el desarrollo de las capacidades bilingües de los alumnos en ambas lenguas, sin olvidar el aprendizaje de una lengua extranjera. Y todo ello sin menoscabo de los conocimientos y de la formación de los alumnos. Como muestra de los avances, tenemos los siguientes datos:

a) El éxito y el prestigio de la escolarización en un modelo (inicialmente pensado para alumnos cuya L1 era el euskera) bi/ plurilingüe que utiliza el euskera como lengua vehicular de todos aprendizajes $^{(8)}$ se ha traducido en un progresivo incremento de la matrícula en ese modelo, al contrario de lo que ha sucedido en los modelos que sólo utilizan el euskera durante algunas horas o en algunas asignaturas.

b) La progresiva matrícula de los alumnos con L1 diferente del euskera en los modelos de escolarización total en euskera ha dado lugar a un proceso de inmersión lingüística para los alumnos que tienen el euskera como L2.

c) La progresiva bilingüización vasco-castellano de la sociedad. Si observamos los datos que nos proporciona la VI Encuesta Sociolingüística (2016), vemos que un 71,4\% de los jóvenes de entre 16 y 24 años conocen el euskera (imagen 5).

d) En la CAE, el $65,92 \%$ de los alumnos matriculados en educación no universitaria durante el curso 2020-2021 realiza sus estudios íntegramente en euskera, y el 17,38\% lo hace de forma parcial (vascoespañol) ${ }^{(9)}$. En el caso de la universidad pública, en la CAE, el 53,9\% de los alumnos se matricularon para realizar sus estudios íntegramente en euskera en el curso 2020-2021(10).

e) La tasa de escolaridad a los 2 años es del 94\% y a los 5 años del 100\% (EUSTAT, 2019(11)). La tasa de fracaso escolar en la CAE fue del 6,9\% el 2018 (frente al 17,9\% del Estado español).

(8) A excepción de las asignaturas de lengua castellana y lengua extranjera.

(9) Fuente: Estadísticas del sistema educativo. Departamento de Educación del Gobierno Vasco.

(10) Fuente: Campusa: https://www.ehu.eus/es/-/34.000-estudiantes-de-grado-comienzan-estosd\%C3\%ADas-las-clases-del-curso-2020-2021-en-la-upv/ehu

(11) https://www.eustat.eus/elementos/ele0002900/TasasdeescolaridadenlaCAdeEuskadiporedadyniveldeensenanza201718/tbl0002915c.html 
Imagen 5. Evolución en \% de la población vascohablante por grupos de edad en la CAE de 1991 al 2016
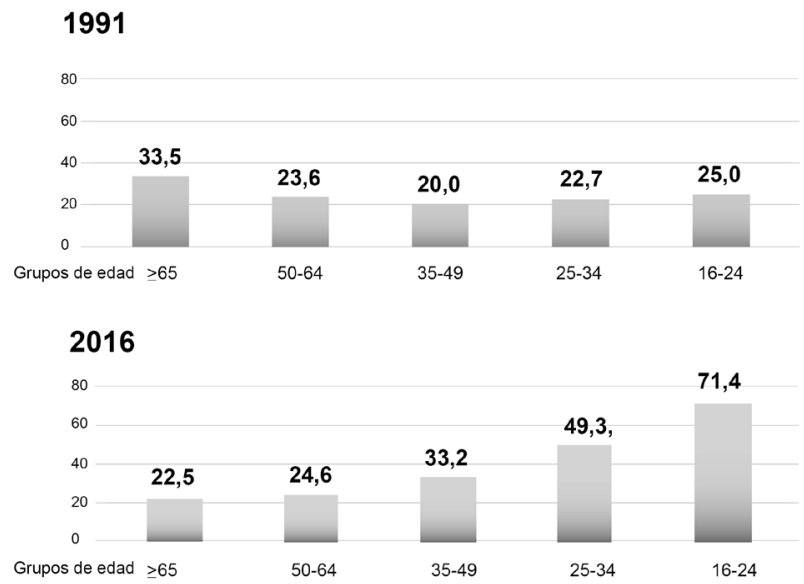

(Fuente: Gobierno Vasco, 2016: 15)

Los logros del proceso de revitalización lingüística han sido evidentes, pero todavía queda mucho camino que recorrer. No todo se ha conseguido. Los tiempos cambian y también las sociedades, y las lenguas no son ajenas a esos cambios. Si bien la mayoría de las sociedades son plurilingües por naturaleza, el estatus de las lenguas que en ella se hablan no siempre es similar. El abanico de posibilidades y oportunidades que tiene la lengua hegemónica y mayoritaria para ser utilizada y desarrollada en la sociedad, es muy superior al que tienen las lenguas minorizadas, lo cual supone siempre una ventaja para el buen dominio de la lengua mayoritaria, pues como muestran numerosas investigaciones (Cenoz, 2009; Manterola et al., 2013; Larringan et al., 2015), aún cuando la educación bilingüe o plurilingüe garantiza el aprendizaje de la lengua originaria y de una lengua extranjera; la lengua hegemónica, por su accesibilidad y disponibilidad, siempre tiene ventaja.

Ese es un reto al que las lenguas originarias y minorizadas tienen que hacer frente, pero no es el único, existen dos más. Por un lado, tenemos la creciente presión del inglés que ha desbancado de los programas escolares de muchos países al resto de las lenguas extranjeras. En el caso del castellano, por ejemplo, esa presión condujo a una transformación de la oferta educativa, de manera que las regiones monolingües se han apresurado a reconvertir sus modelos educativos monolingües en castellano en modelos educativos bilingües castellano-ingles (paradójicamente, sin que por ello haya cambiado su visión monolingüe del mundo); el inglés ha cobrado un inusitado protagonismo y de ser la asignatura de lengua 
extranjera ha pasado a ser una de las lenguas vehiculares de los aprendizajes en muchas escuelas y universidades. En el caso de la $C A E$, la educación bilingüe vascocastellano (o plurilingüe vasco-castellano-lengua extranjera, si tenemos en cuenta que la lengua extranjera también ha estado siempre presente en la educación habitualmente denominada "bilingüe") era una realidad mucho antes de esa trasformación, pero también esas escuelas han sido objeto de una "reconversión" por lo que muchas adoptaron en los últimos años un modelo de educación trilingüe (vasco-castellano-ingles) en el que el inglés aumentó su protagonismo y las tres lenguas se utilizan como lenguas vehiculares de los aprendizajes. Está por conocerse, no obstante, el efecto que ese modelo tendrá sobre el uso del euskera, especialmente, si esta lengua ya de por sí minorizada se ve desplazada por el inglés en las etapas más tempranas de la escolarización.

Por otro lado, no podemos obviar el gran cambio social y sociolingüístico producido por las migraciones. Ciudades y pueblos en los que antes predominaba cierta homogeneidad o uniformidad, se han convertido en un crisol de culturas y de lenguas. En consecuencia, en nuestras escuelas, además del vasco y del castellano, podemos encontrar en la misma aula una gran variedad de lenguas diferentes, de familias procedentes de todo el mundo. La incorporación de esos alumnos a la educación plurilingüe vasco-español-inglés en sus diferentes modalidades nos obliga a reforzar y repensar las estrategias de revitalización lingüística de la lengua originaria sin dejar fuera del proceso esta diversidad lingüística y a sus lenguas maternas, y a buscar nuevas estrategias didácticas para los procesos de enseñanzaaprendizaje de las lenguas, que como han mostrado Díaz de Gereñu y GarcíaAzkoaga (2016) y Manterola et al. (2012), puede ser exitoso incluso en el caso de los alumnos que se incorporan a la escuela con lenguas extranjeras diferentes a las de la comunidad de acogida.

\section{La diversidad lingüística en la escuela}

Gracias al Programa Educación para Todos 2000-2015 (UNESCO, 2015), en la mayoría de los países de América Latina y el Caribe, durante ese período, aumentó la inversión pública realizada en educación (situándose, según los países, entre un 3\% a 15\%), lo que a pesar de las diferencias, en líneas generales, permitió mejorar el acceso a la educación. Uno de los indicadores empleados para realizar el seguimiento al programa es el IDE (Índice de Desarrollo de Educación para Todos), el cual mide cuatro de los seis objetivos de la EPT: la educación primaria universal, la alfabetización de adultos, la igualdad de género y la paridad, y la calidad educativa (los otros dos objetivos son: cuidado de la primera infancia y habilidades de jóvenes y adultos). Como vemos, ninguno de esos objetivos hace referencia a la diversidad cultural y lingüística, que sin duda, es fundamental para la alfabetización y para una educación equitativa y de calidad. En el informe de seguimiento (UNESCO, 2015) solo encontramos una referencia a los grupos indígenas para ejemplificar 
las políticas implementadas destinadas a ampliar las oportunidades educativas; según el informe:

"Desde la década de 1990, en la región ha aumentado la ayuda internacional que apoya los programas de educación intercultural y bilingüe. En Guatemala, las reformas incluyeron la Ley de Idiomas Nacionales, en 2003, que exigió respeto por las lenguas indígenas, además de la creación de un Viceministerio de Educación Intercultural Bilingüe, también en 2003, el cual estableció institutos para la formación de docentes bilingües y educación bilingüe a nivel de primaria. Estas medidas tuvieron un impacto importante en un contexto donde los niveles de educación inicial eran bajos y también muchos indígenas no hablaban el idioma dominante." (UNESCO, 2015: 10)

Se habla de ampliar oportunidades educativas, promover la igualdad de género, mejorar la formación de los docentes y la calidad de los programas educativos, pero no se realizan más alusiones a las lenguas. Además, cuando se aborda la cuestión de la alfabetización, se dice explícitamente:

"Entre las encuestas más precisas sobre alfabetización está la encuesta Skills towards Employment and Productivity (STEP), presentada en 2010 por el Banco Mundial en 13 países de ingresos medios, y el Programa de Evaluación y Seguimiento de la Alfabetización de la UNESCO (LAMP, por sus siglas en inglés), que define tres niveles de dominio de la alfabetización.

En el Estado Plurinacional de Bolivia y Colombia, las encuestas STEP mostraron una clara relación entre el dominio y la cantidad de lectura requerida por la ocupación de los encuestados. Para Paraguay, los resultados del LAMP muestran que los adultos de las zonas rurales tienen habilidades de lectura en prosa más bajas que las que tienen los habitantes urbanos. Sólo el 9\% de los adultos rurales alcanzó el nivel más alto de habilidades de lectura en prosa en comparación con el 20\% de los adultos de zonas urbanas, posiblemente porque los habitantes de las zonas rurales identifican menos el español como lengua materna, de lo que lo hacen los residentes urbanos. Al hacer referencia a términos de habilidades numéricas, no existe tal diferencia entre las zonas rurales y urbanas." (UNESCO, 2015: 6-7).

Está claro que se trata de planteamientos que emanan de visiones del mundo monolingües y propias de lenguas dominantes o hegemónicas. ¿Cómo es posible que se utilice la misma herramienta para medir las competencias de alfabetización de los hablantes sin tener en cuenta cuál es su L1 (lengua materna o familiar)?, ¿cómo se pretende medir la competencia de alfabetización en una lengua 
con una herramienta pensada para otra?, ¿por qué no se realizan las mediciones en las lenguas maternas o familiares?, ¿qué oportunidades de ser alfabetizados en sus lenguas maternas tienen los integrantes de las comunidades indígenas para desarrollar competencias en esas lenguas?

Fijémonos ahora en los Objetivos de Desarrollo Sostenible. El número cuatro es el de la Educación (ODS4), que consiste en "garantizar una educación inclusiva, equitativa y de calidad y promover oportunidades de aprendizaje durante toda la vida para todos". En la Declaración de Incheon y Marco de Acción para la Realización del Objetivo de Desarrollo Sostenible $4(2016)^{(12)}$, pone el acento en la calidad de la educación, y aunque brevemente, también incluye una referencia al plurilingüismo cuando habla de la meta 4.1:

"En contextos plurilingües, siempre que sea posible y teniendo en consideración las distintas realidades, capacidades y políticas nacionales y sub-nacionales, se deberán promover el aprendizaje y la enseñanza en la primera lengua o la hablada en el hogar. Habida cuenta del aumento dela interdependencia social, ambiental y económica en todo el mundo, también se recomienda ofrecer al menos una lengua extranjera como asignatura." (UNESCO, 2016: 37).

De su correspondiente estrategia indicativa:

"En los contextos plurilingües, promover la educación bilingüey plurilingüe, comenzando con el aprendizaje temprano en la primera lengua de los niños o la hablada en casa." (UNESCO, 216: 38).

Y del indicador que se propone:

"Porcentaje de estudiantes de educación primaria cuya primera lengua o lengua que utilizan en el hogar corresponde a la lengua de instrucción." (UNESCO, 216: 78).

La cuestión es: ¿cuáles son las claves de esa presencia o de ese plurilingüismo?, ¿en qué medida se aborda la cuestión de las lenguas indígenas?

El informe de la ONU de 2019 sobre el seguimiento de los ODS y la Agenda 2030(13) nos presenta cifras que todavía resultan desoladoras: 617 millones de niños y adolescentes no alcanzaron el nivel mínimo de competencia en lectura y matemáticas, lo que en palabras de la ONU significa una "crisis mundial de

(12) https://unesdoc.unesco.org/ark:/48223/pf0000245656spa 
enseñanza" (SDG Reports, 2019: 30); en el mundo 750 millones de adultos son analfabetos, de ellos dos tercios son mujeres; 1 de cada 5 niños de entre 6 y 17 años no asiste a la escuela: las niñas que no asisten a la escuela son un $27 \%$ más que los niños en el caso de Asia; más de la mitad de las escuelas en el África subsahariana no tienen acceso a servicios básicos de agua potable, instalaciones para lavarse las manos, internet, computadoras; en muchos casos, ni siquiera cuentan con docentes cualificados. La educación infantil de calidad se revela como fundamental en la posterior escolarización de los niños, pero en el mundo solo el $69 \%$ participa de una enseñanza infantil organizada (y las diferencias entre países son enormes, pues van desde el $7 \%$ hasta el $100 \%$ ).

En ese informe tampoco se hace ninguna referencia a las lenguas. Sin embargo, sabemos que una de las causas de la no asistencia a la escuela y del fracaso escolar es el hecho de que en la escuela se privilegia la lengua hegemónica como lengua vehicular de los conocimientos y se desplaza e incluso menosprecia a la lengua autóctona. Eso trae consigo, además, modelos de conocimiento descontextualizados y poco ajustados a la realidad y a la cosmovisión de las comunidades indígenas. No tienen en cuenta que las lenguas indígenas pueden servir de herramienta para adecuarse a los nuevos tiempos, pues como señalan Mendizabal y Uranga (2020), uno de los objetivos debe ser poner de relieve una visión propia del mundo y dar prestigio a esa cosmovisión, y eso se logra utilizando la lengua indígena como herramienta. Al fin y al cabo, como subrayan Larrañaga y Barreña (2020), incorporarse al mundo moderno no es renunciar al conocimiento heredado, pues lengua, cultura e identidad son claves para la consecución de un desarrollo equilibrado, digno y justo; recordemos, como dicen Skutnabb-Kangas et al. (2003), que son esas lenguas las que contienen las claves para la supervivencia, y por lo tanto, del desarrollo. Por eso es importante que las lenguas originarias estén presentes en la educación.

Hay países en los que existe una única lengua oficial, pero eso no significa que los hablantes sean monolingües o que no hayan comunidades de hablantes de otras lenguas, pues como señalan Garcia-Azkoaga y Pérez-Caurel (2019) en muchos casos el multilingüismo es la forma natural de relacionarse, y la herramienta que permite adaptarse al entorno, y que va más allá de imposiciones y regulaciones legales. Sin embargo, es habitual que una lengua sea mayoritaria y hegemónica y otra u otras se marginen y se conviertan en lenguas minorizadas. Una de las principales consecuencias que tiene ese desequilibrio es que deja fuera de la escuela a las lenguas autóctonas para dar prioridad a una educación en la lengua oficial hegemónica, muchas veces, por efecto de procesos coloniales.

A lo largo del mundo se han llevado a la práctica diferentes experiencias de educación bi/plurilingüe. En el caso de América Latina, por ejemplo, la 
Educación Intercultural Bilingüe (EIB) surgió para dar solución a ese desequilibrio y buscar soluciones para contribuir a la recuperación o al reforzamiento de la lengua originaria, según los casos (López y Kuper, 2000), a la vez que se garantizaba el aprendizaje de la lengua oficial hegemónica. No obstante, los avances realizados no siempre fueron los deseados, principalmente, porque las lenguas originarias no contaron con el suficiente reconocimiento ni fueron suficientemente prestigiadas ni reivindicadas por sus propios hablantes, y porque una educación bilingüe o plurilingüe de calidad requiere de un compromiso curricular y formativo que no se limite exclusivamente a los primeros años de la escolarización. Una apuesta por avanzar en la mejora de la EIB, es, por ejemplo, el Plan Nacional de EIB al 2021 en Perú (MINEDU, 2016).

La práctica de utilizar la lengua materna en el primer contacto de los niños con la escuela está bastante extendida también en algunos lugares de África, pero frecuentemente, por causas muy diferentes, se limita al uso oral de esas lenguas y no se extienden como debieran al aprendizaje de la lecto-escritura, a pesar de que estudios como el de Ouane y Glanz (2010), entre otros, hacen hincapié en las ventajas del plurilingüismo y en la necesidad de abordar el tema de la educación plurilingüe en África.

Aunque, como nos muestra Cummins (2019), no todas son igual de efectivas ni buscan los mismos objetivos, en el mundo hay numerosas experiencias de educación plurilingüe basadas en la lengua materna que han demostrado ser exitosas, incluso, cuando se trata de una educación inmersiva en lengua minorizada. Este es el caso del vasco, que no es obstáculo para que los alumnos aprendan sin problemas la lengua hegemónica (Garcia-Azkoaga e Idiazabal, 2015). Aunque las circunstancias y el contexto son diferentes, también podemos ver iniciativas plurilingües exitosas en los ejemplos que nos proporciona Benson (2019) sobre educación plurilingüe en lengua materna, por ejemplo, en el caso del wolof y el pulaar (lenguas minorizadas o no dominantes) junto al francés (lengua hegemónica) en Senegal, el uso de cinco lenguas indígenas no dominantes (brao, bunong, kavet, kreung y tampuen) junto al jemer (lengua dominante) en educación primaria en Camboya, o la educación primaria basada en la lengua materna en Etiopía.

En cualquier caso, como recuerdan Idiazabal (2017) y Garcia-Azkoaga (2019), a la hora de abordar políticas plurilingües, especialmente en la educación, no podemos obviar el papel que juegan la administración, los propios hablantes y las familias, pues solo su voluntad y su empoderamiento puede accionar el motor de la educación plurilingüe y de la revitalización lingüística. El papel de la escuela, así como el de todos los agentes relacionados con ella es fundamental, pero lo realmente importante es la consecución de lo que Benson (2019) Ilama 
un "habitus plurilingüe", una forma plurilingüe de concebir el mundo, pues no en vano el punto de vista crea el objeto. La autora subraya que el mérito del desarrollo curricular plurilingüe de las escuelas vascas es, precisamente, el haber creado un "hábito multilingüe", es decir, una forma de concebir y llevar a cabo el plurilingüismo, hábito que considera esencial para afrontar el reto de una educación plurilingüe.

\section{Conclusiones}

La actividad del lenguaje es inherente a la actividad humana y su funcionamiento es universal. Sea cual sea la lengua o el código que utilicemos la actividad verbal es la base de las interacciones humanas, sirve para transmitir y compartir conocimientos, para construir las redes sociales y para transformar la sociedad; además, dado que esa actividad verbal se materializa en lenguas o idiomas concretos, la forma en que se desarrollará la actividad humana dependerá también de la lengua utilizada. En el caso de las lenguas indígenas y de las lenguas que han sido minorizadas a causa de los procesos coloniales y los avatares históricos, esas lenguas han sido sustituidas por otras lenguas hegemónicas que privaron a aquellas de su universo de actividad verbal y modificaron así su actividad humana, restando a los individuos una parte de sus derechos. Cuando un hablante ve limitadas las posibilidades de comunicarse en su propia lengua, la actividad verbal y social también se resiente. Aunque el funcionamiento del lenguaje y de la comunicación puedan considerarse universales, la actividad verbal reposa en la utilización de un código conocido y compartido por la comunidad, que es generador de intercambio y de conocimiento. Por lo tanto, no es lo mismo construir y desarrollar el conocimiento en la lengua propia, que en una lengua impuesta que ignora y menosprecia la existencia de las demás lenguas.

Llevar a buen fin la Agenda 2030 de los Objetivos de Desarrollo Sostenible implica actuar en diversas facetas de la actividad humana con el objeto de incidir o mediar de alguna forma en esa actividad. Esa mediación se sustenta en las lenguas de los propios hablantes y de las propias comunidades a las que van dirigidas las acciones que se llevan a cabo en la promoción de la citada Agenda, por lo que el objetivo de la diversidad lingüística o las políticas plurilingües debería estar presente de forma transversal en todas esas acciones.

El respeto a la diversidad lingüística comienza con el reconocimiento de las lenguas indígenas y minorizadas, y de los derechos de sus hablantes, como el derecho a utilizar su propia lengua autóctona y el derecho a ser alfabetizado y educado en esa lengua autóctona. Conseguirlo, no obstante, supone muchas veces abordar procesos de revitalización y planificación 
lingüística que ayuden en primer lugar a prestigiar las lenguas, y sobre todo, articular un sistema educativo plurilingüe de calidad; para eso es necesaria una voluntad clara por parte de los gobernantes. Con la asunción de una realidad plurilingüe que en un Estado no tiene por qué estar contrapuesta al uso como lengua franca o lengua común de una lengua oficial compartida para todos los territorios, y con una visión estratégica de cambio social a largo plazo, contemplando la revitalización lingüística como una inversión para el desarrollo de las comunidades y de los Estados y, por lo tanto, como un beneficio para todos.

Sin duda, la escuela es la columna vertebral del desarrollo y la pieza esencial para conseguir un mundo mejor y más justo para todos, pero garantizar una educación inclusiva y de calidad implica necesariamente abordar con seriedad y rigor una educación plurilingüe que pivote en torno a la lengua materna y que permita a los alumnos desarrollar plenas capacidades en su lengua familiar, en la lengua oficial hegemónica $y$, al menos, en una lengua extranjera. En este conglomerado plurilingüe tampoco podemos olvidar la presencia en la escuela de las lenguas de inmigración, que aunque no sean las lenguas vehiculares de los aprendizajes formales son un pilar fundamental de los aprendizajes no formales en el seno de la familia. Por un lado, sabemos que los aprendizajes o las capacidades desarrolladas durante el aprendizaje de una lengua son transferibles y utilizables en el aprendizaje de las otras lenguas, así que la estrategia de ignorar una lengua a favor de otra no tiene ningún sentido. Lo que se trata es de aprovechar las ventajas de ese conocimiento para ampliar y adquirir conocimientos nuevos. La verdadera cuestión es profundizar en el estudio de las herramientas pedagógicas que favorezcan el desarrollo de las capacidades plurilingües. Por otro lado, un desarrollo curricular plurilingüe que vaya más allá de la educación infantil, puede facilitar el acceso a los puestos de trabajo y al desarrollo profesional. Junto con la escuela, también es esencial la alfabetización de los adultos en sus propias lenguas maternas, pues de ello depende en gran medida las posibilidades de formar a los profesionales que necesita la escuela plurilingüe. De ello depende también el poder desarrollar una escuela insertada en la propia comunidad, que explore el conocimiento relevante para su propio contexto; una escuela adaptada a su entorno, a sus necesidades sociales y de desarrollo, cercana y aprehensible por la propia comunidad.

Son todas estas medidas necesarias para garantizar los derechos de los pueblos indígenas y fomentar su desarrollo, pero por supuesto, requieren superar los prejuicios infundados sobre el fomento del multilingüismo en la escuela, así como que las acciones en favor de las lenguas indígenas no se queden reducidas a la conmemoración de un único año. 


\section{Referencias}

BAZTARRIKA, P. (2019). El modelo vasco de revitalización lingüística y desarrollo sostenible. En I. Idiazabal \& M. Pérez-Caurel (coord.). Diversidad Lingüística, Lenguas Minorizadas y Desarrollo Sostenible. Bilbao: Servicio Editorial de la UPV/EHU. pp. 239-262. Recuperado de https://addi.ehu.es/handle/10810/34728 (Enero de 2020).

BENSON, C. (2019). The importance of learners'own languages in achieving Sustainable Development Goal Four (Ethiopia and Cambodia). En I. Idiazabal \& M. Pérez-Caurel (coord.). Diversidad Lingüística, Lenguas Minorizadas y Desarrollo Sostenible. Bilbao: Servicio Editorial de la UPV/EHU. 239-262. Recuperado de https:// addi.ehu.es/handle/10810/34728 (Enero de 2020).

CENOZ, J. (2009). Towards Multilingual Education Basque Educational Research from an International Perspective. Clevedon: Multilingual Matters.

CUMMINS, J. (2019). Should schools undermine or sustain Multilingualism? An analysis of Theory, research, and pedagogical Practice, Sustainable Multilingualism, 15. pp. 1-26. https://doi.org/10.2478/sm-2019-0011.

DIAZ DE GEREÑU, L. \& GARCIA-AZKOAGA, I.M. (2016).Aprendiendo dos lenguas nuevas. Los cuentos orales de los niños inmigrantes. Lengua y migración/Language and migration, 8:1, pp. 29-49.

GARCIA-AZKOAGA, I.M. (2019). Didáctica de las lenguas y educación bi/plurilingüe: algunos retos para la EIB, Enunciación, Vol. 24 (1), 45-60. DOI: 10.14483/issn.22486798. Recuperado de https://revistas.udistrital.edu.co/index.php/enunc/article/ view/13263/14718 (Octubre de 2019).

GARCIA-AZKOAGA, I.M. \& IDIAZABAL, I. (eds.) (2015). Para una ingeniería didáctica de la educación plurilingüe. Bilbao: Universidad del País Vasco.

GARCIA-AZKOAGA, I.M. \& PÉREZ-CAUREL, M. (2019). La comunidad de habla baribá: algunas reflexiones sobre el multilingüismo y la educación plurilingüe, Sustainable Multilingualism, 15. pp. 105-128. https://doi.org/10.2478/sm-201900161.Recperado de https://hdl.handle.net/20.500.12259/102030 (Octubre de 2019).

GENERALITAT DE CATALUNYA (2019). EMMARCA'T, Departament de Cultura. Recuperado de https://lengua.gencat.cat/ca/serveis/empresa/emmarcat/ (Abril de 2020).

GOBIERNO DE ESPAÑA (1978). Constitución Española. BOE no 311, de 29/12/1978. Recuperado de https://www.boe.es/buscar/act.php?id=BOE-A-1978-31229 (Octubre de 2019). 
GOBIERNO DE ESPAÑA (1979). Ley Orgánica, de 18 de diciembre, de Estatuto de Autonomía para el País Vasco. BOE no 306, de 22 de diciembre de 1979. Recuperado de https://www.boe.es/buscar/act.php?id=BOE-A-197930177\& $=19791222 \& \mathrm{tn}=2$ (Octubre de 2019).

GOBIERNO DE ESPAÑA (1982). Ley Orgánica 13/1982, de 10 de agosto, de reintegración y amejoramiento del Régimen Foral de Navarra. BOE no 195, de 16 de agosto de 1982. Recuperado dehttps://www.boe.es/buscar/doc.php?id=BOE-A1982-20824(Octubre de 2019).

GOBIERNO VASCO (1982), Ley 10/1982, de 24 de Noviembre, básica de normalización del uso del Euskera. BOPV n॰160 de 16de diciembre de 1982. Recuperado de https:// www.euskadi.eus/y22-bopv/eu/bopv2/datos/1982/12/8201955e.shtml(Octubre de 2019).

GOBIERNO VASCO (2016). VI Encuesta Sociolingüística. Vitoria-Gasteiz: Servicio Editorial del Gobierno Vasco. Recuperado de https://www.google.com/search?clie nt=firefoxbd\&q=Vl+encuesta+sociolingusitica+3016\#(Octubre de 2019).

IDIAZABAL, I. (2017). ¿Qué significa la escuela bilingüe para lenguas minorizadas como el nasa yuwe o el euskera? [Monografía]. Onomázein: Las lenguas amerindias en Iberoamérica: retos para el siglo XXI, 138-152. DOI: 10.7764/onomazein. amerindias.08.

INSTITUTO VASCO DE ESTADÍSTICA (EUSTAT) (2019).Tasas de escolaridad en la C.A. de Euskadi por edad y nivel de enseñanza (\%) 2017/18. Recuperado de https:// www.eustat.eus/elementos/ele0002900/TasasdeescolaridadenlaCAdeEuskadipor edadyniveldeensenanza201718/tbl0002915c.html (Octubre de 2019).

LARRAÑAGA, J. \& BARREÑA, A. (en prensa). Hizkuntza-lankidetza: oraina eta geroa. En L. Diaz de Gereñu, L., I.M. García-Azkoaga e I. Manterola: Euskara oinarri eta eleaniztas una helburu. Bilbao: Servicio Editorial de la UPV/EHU.

LARRINGAN, L.M., IDIAZABAL, I. y GARCÍA-AZKOAGA, I.M (2015). Cartas al director escritas en euskara y castellano por jóvenes bilingües. Transferencias y destrezas textuales y discursivas. En I.M. García-Azkoaga e I. Idiazabal (eds.), Para una ingeniería didáctica de la educación plurilingüe, pp. 147-172. Bilbao, España: UPV/ EHU.

LÓPEZ, L.E. \& KÜPER, W. (1999). La educación intercultural bilingüe en América Latina: balance y perspectivas. Revista Iberoamericana de Educación, 20, pp. 1-45.

MANTEROLA, I., ALMGREN, M. \& IDIAZABAL, I. (2012). El aprendizaje del euskera en niños de origen inmigrante: influencia de la escuela y del entorno, Esquisses pour une école plurilingüe: contextualiser nos réflexions sociodidactiques. En M. Totozani, S. Tomé, M. Rispail y C. Jeannot (eds.), pp. 199-217. Paris: L'Harmattan. 
MANTEROLA, I., ALMGREN, M. \& IDIAZABAL, I. (2013). Basque L2 development in immersion school settings. International Journal of Bilingualism, 17(3), pp. 375-391.

MARINOTTI, J.-P. (2016). Final Report Symposium on Language and the Sustainable Development Goals. New York: United Nations, Report Study Group on Language and the UN. Recuperado de http://www.languageandtheun.org/documents.html (Septiembre de 2019).

MARINOTTI, J.-P. (2017). Final Report Symposium on Language, the Sustainable Development Goals and Vulnerable Populations. New York: United Nations, Report Study Group on Language and the UN. Recuperado de http://www. languageandtheun.org/documents.html (Septiembre de 2019).

MARTÍ, F.; ORTEGA, P.;IDIAZABAL, I; BARREÑA, A.; JUARISTI, P.; JUNYENT, C.; URANGA, B. \& AMORRORTU, E. (eds.) (2006). Palabras y mundos. Informe sobre las lenguas del mundo. Barcelona: Icaria.

MENDIZABAL, M. \& URANGA, B. (en prensa). Munduko hizkuntza-aniztasunetik euskarara; ekologietatik begirada. En L. Díaz de Gereñu, L., I.M. García-Azkoaga e I. Manterola (eds.): Euskara oinarri eta eleaniztas una helburu. Bilbao: Servicio Editorial de la UPV/EHU.

MINEDU (2016). Plan Nacional de Educación Intercultural Bilingüeal 2021. Recuperado de http://www.minedu.gob.pe/campanias/pdf/eib-planes/rm-629-2016-mineduplan-nacional-eib.pdf (Noviembre de 2020).

NACIONES UNIDAS(2007). Declaración de las Naciones Unidas sobre los derechos de los pueblos indígenas. Recuperado de https://www.un.org/development/ desa/indigenous-peoples-es/declaracion-sobre-los-derechos-de-los-pueblosindigenas.html (Octubre 2019).

NACIONES UNIDAS (2014) Documento final sobre la Conferencia Mundial sobre los Pueblos Indígenas. Recuperado de https://www.un.org/es/ga/69/meetings/ indigenous/documents.shtml (Octubre de 2019).

NACIONES UNIDAS (2015). Agenda 2030 de los Objetivos de Desarrollo Sostenible (ODS). Recuperado de https://www.un.org/sustainabledevelopment/es/2015/09/ la-asamblea-general-adopta-la-agenda-2030-para-el-desarrollo-sostenible/ (Octubre de 2019)

NACIONES UNIDAS (2019). Informe de los Objetivos de Desarrollo Sostenible 2019, Departamento de Asuntos Económicos y Sociales (DESA), New York: United Nations Publications. Recuperado de: https://www.un.org/sustainabledevelopment/es/ development-agenda/ (Octubre de 2019). 
ORGANIZACIÓN DE LOS ESTADOS AMERICANOS (2016). Declaración americana sobre los derechos de los pueblos indígenas. Recuperado de https://www.oas.org/es/ sadye/documentos/res-2888-16-es.pdf (Noviembre de 2020).

ORGANIZACIÓN INTERNACIONAL DEL TRABAJO (1989). Convenio sobre pueblos indígenas y tribales de la Organización Internacional del Trabajo (1989) ilo.org/dyn/normlex/es/f?p=NORMLEXPUB:12100:0::NO::P12100 ILO CODE:C169.

OUANE, A. \& GLANZ, C. (2010). Why and how Africa should invest in African languages and multilingual education: an evidence- and practice-based policy advocacy brief. UNESCO Institute for Lifelong Learning. Hamburg: Germany. Recuperado de http:// www0.sun.ac.za/taalsentrum/assets/files/ML\%20Afr\%20Lang\%20\&\%20Cost.pdf (Septiembre de 2019).

SKUTNABB-KANGAS, T., MAFFI, L. \& HARMON, D. (2003). Sharing a World of Difference. The earth's linguistic, cultural and biological diversity. Paris: UNESCO/ WWF/Terralingua. Recuperado de https://unesdoc.unesco.org/ ark:/48223/ pf0000132384 (Febrero 2020).

UNITED NATIONS EDUCATIONAL SCIENTIFIC AND CULTURAL ORGANISATION [UNESCO] (2016). Declaración de Incheon y Marco de Acción ODS 4 - Educación 2030. Recuperado de https://unesdoc.unesco.org/ark:/48223/pf0000245656 spa(Marzo de 2020).

UNITED NATIONS EDUCATIONAL SCIENTIFIC AND CULTURAL ORGANISATION [UNESCO] (1953). Empleo de las lenguas vernáculas en la enseñanza. Paris: UNESCO. Recuperado de https://unesdoc.unesco.org/ark:/48223/pf0000131583 (Octubre de 2019).

UNITED NATIONS EDUCATIONAL SCIENTIFIC AND CULTURAL ORGANISATION [UNESCO] (2015). Panorama regional américa latina y el caribe informe mundial de seguimiento de la ept 2015informe mundial de seguimiento de la EPT Recuperado de https://unesdoc.unesco.org/ark:/48223/pf0000232836spa (Febrero de 2020).

UNIVERSIDAD DEL PAÍS VASCO (2019). EHU agenda 2030 por el desarrollo sostenible. Bilbao: Servicio Editorial de la UPV/EHU. 